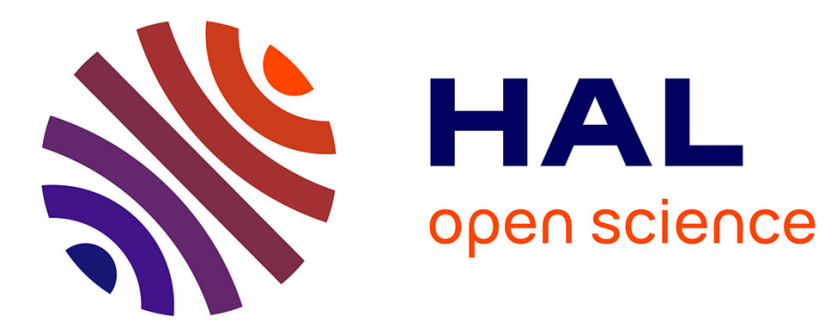

\title{
Le maser à hydrogène en régime transitoire
}

Claude Audoin

\section{To cite this version:}

Claude Audoin. Le maser à hydrogène en régime transitoire. Revue de Physique Appliquée, 1967, 2 (4), pp.309-320. 10.1051/rphysap:0196700204030900 . jpa-00242810

\section{HAL Id: jpa-00242810 https://hal.science/jpa-00242810}

Submitted on 1 Jan 1967

HAL is a multi-disciplinary open access archive for the deposit and dissemination of scientific research documents, whether they are published or not. The documents may come from teaching and research institutions in France or abroad, or from public or private research centers.
L'archive ouverte pluridisciplinaire HAL, est destinée au dépôt et à la diffusion de documents scientifiques de niveau recherche, publiés ou non, émanant des établissements d'enseignement et de recherche français ou étrangers, des laboratoires publics ou privés. 


\title{
LE MASER A HYDROGÈNE EN RÉGIME TRANSITOIRE ( $\left.{ }^{1}\right)$
}

\author{
Par Glaude AUDOIN ( ${ }^{2}$ ), \\ Section d'Orsay du Laboratoire de l'Horloge Atomique, \\ Institut d'Électronique Fondamentale, Faculté des Sciences, Orsay.
}

\begin{abstract}
Résumé. - Nous montrons que l'étude du comportement dynamique du maser à hydrogène, au voisinage de la valeur d'équilibre du niveau d'oscillation, permet la mesure directe du temps de relaxation $T_{1}$ des atomes d'hydrogène, ainsi que celle de l'amplitude du champ électromagnétique dans la cavité résonnante.

L'effet d'un champ magnétique statique inhomogène couplant entre eux les sous-niveaux Zeeman $F=1$ est examiné en détail. Nous calculons l'erreur systématique sur la mesure de $T_{1}$ résultant de la présence d'un tel champ magnétique et nous montrons qu'elle peut être rendue facilement négligeable.
\end{abstract}

Abstract. - We show here that the study of the dynamic behaviour of the atomic hydrogen maser near the equilibrium oscillation level allows us to measure directly the relaxation time $T_{1}$ of the hydrogen atoms as well as the magnitude of the electromagnetic field in the resonant cavity.

The effect of a static inhomogeneous magnetic field coupling the Zeeman sub-levels $F=1$ between them is discussed in detail. We calculate the systematic error on the measurement of $T_{1}$ resulting from the presence of such a magnetic field and show that this error can be easily made negligible.

Introduction. - L'oscillateur à hydrogène atomique a été mis au point en 1960, à l'Université de Harvard, dans l'équipe du Professeur Ramsey [1].

Il utilise la transition de structure hyperfine $F=1, M=0 \rightarrow F=0, M=0$, de l'atome d'hydrogène à l'état fondamental $1^{2} S^{1 / 2}$.

La fréquence de transition, extrapolée en champ magnétique nul, est, dans l'échelle de temps atomique $A_{1}\left(C_{\mathrm{s}}=9192631770 \mathrm{~Hz}\right)$ [2] :

$$
f_{0}=1420405751,778 \mathrm{~Hz} \pm 0,016 \mathrm{~Hz} \text {. }
$$

La caractéristique essentielle de ce type d'oscillateur est que les atomes restent en interaction avec le champ électromagnétique pendant un intervalle de temps long - quelques dixièmes de seconde - pour compenser la petitesse des éléments de matrice de la transition dipolaire magnétique.

Le confinement des atomes d'hydrogène dans la région centrale d'une cavité résonnante (mode $\mathrm{TE}_{011}$ ) est obtenu en imposant aux atomes d'un jet de pénétrer dans un ballon dont ils s'échappent au bout d'un temps de séjour moyen de $0,5 \mathrm{~s}$ environ. L'état interne des atomes n'est pas sensiblement modifié par les quelque $10^{4}$ chocs sur la paroi du ballon lorsqu'elle est recouverte de fluoro-éthylène ("Téflon »).

(1) Cette étude est subventionnée par le C.N.R.S. et la D.R.M.E.

(2) Cet article recouvre la première partie d'une thèse de Doctorat ès Sciences Physiques soutenue le 30 mai 1967 devant la Faculté des Sciences de Paris. Elle est enregistrée au C.N.R.S. sous le no A.O. 1424.
Si l'on a préalablement enrichi la population du niveau d'énergie supérieur, les atomes peuvent alors fournir de l'énergie au champ électromagnétique par émission stimulée. L'auto-oscillation apparaît lorsque cette énergie compense les pertes par effet Joule dans les parois de la cavité résonnante et les circuits de mesure.

Intérêt de l'étude dynamique du maser à hydrogène. - Jusqu'alors, le maser à hydrogène fonctionnant en auto-oscillateur a surtout été étudié en vue des applications suivantes :

1) Réalisation d'étalons actifs de fréquence [3], [4], [5] dont les performances sont actuellement inégalées;

2) Détection de résonance de particules paramagnétiques introduites dans le gaz d'hydrogène [6].

Dans ces études, le niveau d'oscillation est maintenu stationnaire et on n'a pas - semble-t-il - porté attention au régime transitoire provoqué par une variation brusque des conditions de fonctionnement.

Comme nous le montrons, l'observation du comportement dynamique du niveau d'oscillation, du maser à hydrogène, au voisinage des conditions d'équilibre, rend possible la mesure du temps de relaxation $T_{1}$ des atomes d'hydrogène dans les conditions d'oscillation. Cette mesure est facilitée par l'emploi d'une boucle de réaction qui permet de faire varier le coefficient de surtension de la cavité résonnante, et par là même le niveau d'oscillation dans de grandes limites. Cet artifice expérimental permet également de déter- 
miner, en valeur relative, la densité d'atomes dans le ballon de stockage.

Par l'exploitation de ces nouvelles méthodes de mesure, il est possible de déterminer la section efficace d'échange de spin entre atomes d'hydrogène, ainsi que l'un des paramètres fondamentaux de ce type d'oscillateur : le paramètre q introduit par Ramsey et al. [7].

Les prolongements de cette étude sont également importants si l'on considère que l'effet du bruit de fond, qui détermine la stabilité à court terme de l'oscillateur, peut être calculé comme celui d'une petite perturbation aléatoire du régime stabilisé [8].

Le comportement dynamique du niveau d'oscillation que nous avons étudié a été prévu pour le maser à ammoniac par Grasyuk et Orayevskiy [9], puis par Orayevskiy et Uspenskiy [10] qui ont adopté une démarche différente pour l'établissement des propriétés du milieu actif. A notre connaissance, la vérification expérimentale n'a pas été possible dans ce cas.

\section{L'APPROXIMATION A DEUX NIVEAUX}

Afin de dégager les résultats essentiels de notre étude, nous supposons tout d'abord que les deux niveaux participant à la transition d'horloge $\Delta M=0$ se comportent comme s'ils étaient seuls [11]. La validité de cette hypothèse sera précisée dans la deuxième partie.

\section{I.1. L'opérateur densité des atomes d'hydrogène.} - Nous utilisons le formalisme de l'opérateur densité, qui convient bien à l'étude du comportement d'atomes soumis, à la fois, à une perturbation cohérente et à des perturbations aléatoires.

Le système considéré a la symétrie de révolution autour de l'axe $\mathrm{z}^{\prime} \mathrm{z}$. Le champ magnétique directeur et les largeurs de raies des différentes transitions possibles sont tels que celles-ci sont bien séparées. Les seuls éléments non nuls de la matrice densité, dans la base $|F, M\rangle$, sont donc : $\rho_{F M,} \bar{F}^{\prime} \mathbf{M}^{\prime}$ pour $M=M^{\prime}$ [12].

Par un classement convenable des vecteurs de base, la matrice densité à 4 lignes et à 4 colonnes (pour l'hydrogène $F=0$ ou 1) peut être mise sous la forme de la figure 1 .

Dans l'approximation à deux niveaux, nous faisons

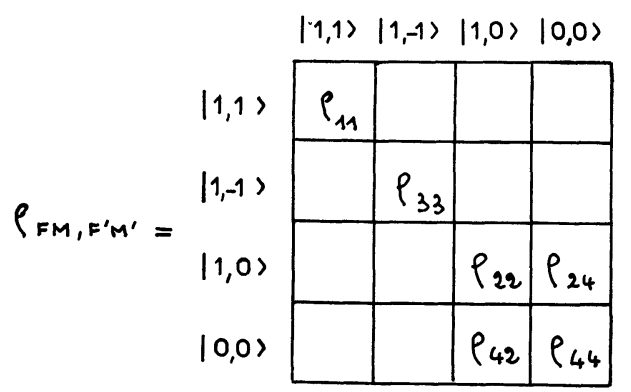

FIG. 1. - Matrice densité

des atomes d'hydrogene dans la base $|F, M\rangle$.

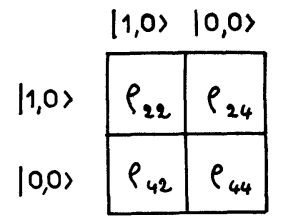

Frg. 2. - Approximation à deux niveaux : matrice densité des atomes d'hydrogène.

l'hypothèse que l'échelon de matrice correspondant aux états $|1,0\rangle$ et $|0,0\rangle$ évolue indépendamment des éléments de matrice $\rho_{11}$ et $\rho_{33}$.

Nous appelons matrice densité dans l'approximation à deux niveaux la matrice à deux lignes et deux colonnes représentée sur la figure 2 .

\section{I.2. Les hypothèses de l'approximation à deux} niveaux. - I.2.1. Gollisions AVEG ÉGHANGE D'ÉLEGTRONS. - Lors d'une interaction en phase gazeuse entre deux atomes d'hydrogène, il peut y avoir échange des électrons (échange de spin) entre les deux atomes [13], [14], [15], [16], [17], [18], [19], ce qui tend à égaliser les populations des états $|1,0\rangle$ et $|0,0\rangle$ et à réduire le moment magnétique des atomes dans un état radiatif.

En conséquence de la structure simple de l'atome d'hydrogène $(I=1 / 2, J=1 / 2)$, l'équation d'évolution des éléments de matrice densité non diagonaux, $\rho_{24}=\langle 1,0|\rho| 0,0\rangle$ et $\rho_{42}=\rho_{24}^{*}$, est linéaire, de la forme [14] :

$$
\left[\frac{\partial}{\partial t} \rho_{\mathbf{2 4}}\right]_{\text {échange }}=-\frac{1}{T_{2}} \rho_{\mathbf{2 4}} .
$$

De même, la différence de population entre les états $|1,0\rangle$ et $|0,0\rangle$ obéit à une équation linéaire simple. F. Grossetête a montré que si l'on considère l'interaction d'échange seule, l'opérateur densité varie selon :

$$
\frac{\partial}{\partial t} \rho=-\frac{1}{T_{1 \mathrm{e}}}\left(\rho-\rho^{\mathrm{e}}\right)
$$

où $\rho^{\mathrm{e}}$ est l'opérateur densité après échange de spin total avec un autre atome d'hydrogène et $T_{1 \mathrm{e}}$ est le temps moyen séparant deux collisions d'échange total.

Une telle collision égalise les populations des niveaux $|1,0\rangle$ et $|0,0\rangle$, et la propriété se conserve en présence de cohérence hyperfine entre les niveaux $M=0 \quad[20]$. Il en résulte $\rho_{22}^{\mathrm{e}}=\rho_{44}^{\mathrm{e}}$ et :

$$
\left[\frac{\partial}{\partial t}\left(\rho_{22}-\rho_{44}\right)\right]_{\text {échange }}=-\frac{1}{T_{1 \mathrm{e}}}\left(\rho_{22}-\rho_{44}\right) .
$$

Nous désignons par $T_{1 \mathrm{e}}$ et $T_{2 \mathrm{e}}$ les constantes de temps $\left(^{3}\right)$ traduisant, macroscopiquement, l'effet des collisions d'échange.

(3) D'une façon générale, l'indice 1 est associé aux éléments de matrice diagonaux, l'indice 2 aux éléments non diagonaux. L'indice littéral caractérise la perturbation. 
I.2.2. Ghocs SUR LA PARoI DU Ballon. - La relaxation sur la paroi du ballon a été étudiée expérimentalement par Berg [17], [19], qui interprète ses résultats en termes d'échange d'électrons entre un atome d'hydrogène et un radical libre formé sur le revêtement du ballon.

Il est généralement admis que les équations les plus simples qui décrivent le résultat de ces expériences sont de la forme [18], [19] :

$$
\left\{\begin{aligned}
{\left[\frac{\partial}{\partial t} \rho_{24}\right]_{\text {paroi }} } & =-\frac{1}{T_{2 \mathrm{p}}} \rho_{24} \\
{\left[\frac{\partial}{\partial t}\left(\rho_{22}-\rho_{44}\right)\right]_{\text {paroi }} } & =-\frac{1}{T_{1 \mathrm{p}}}\left(\rho_{22}-\rho_{44}\right) .
\end{aligned}\right.
$$

Cette hypothèse se trouve confirmée par les résultats de nos expériences où les variations du moment magnétique induit et de la différence de population sont bien décrites par des constantes de temps uniques, respectivement $T_{2}$ et $T_{1}$.

I.2.3. AbSence de Relaxation entre Les sousNiveaux $F=1$. - Nous supposons que le champ magnétique statique appliqué aux atomes est parfaitement homogène de sorte que les sous-niveaux Zeeman ne soient pas couplés entre eux.

Nous lèverons cette restriction dans la deuxième partie, où l'influence de ce type de couplage sera examinée en détail.

I.2.4. É́QuATIONS D'ÉVOLUTION DE L'OPÉRATEUR DENSITÉ SOUS L'INFLUENCE DE LA RELAXATION. - LeS différents processus de relaxation ne sont pas corrélés. Leurs effets se superposent donc, et nous obtenons :

$$
\left\{\begin{array}{r}
{\left[\frac{\partial}{\partial t} \rho_{24}\right]_{\text {relax. }}=-\frac{1}{T_{2}^{\prime \prime}} \rho_{24} ; \quad \frac{1}{T_{2}^{\prime \prime}}=\frac{1}{T_{2 \mathrm{e}}}+\frac{1}{T_{2 \mathrm{p}}}} \\
{\left[\frac{\partial}{\partial t}\left(\rho_{22}-\rho_{44}\right)\right]_{\text {relax. }}=-\frac{1}{T_{1}^{\prime \prime}\left(\rho_{22}-\rho_{44}\right)}} \\
\frac{1}{T_{1}^{\prime \prime}}=\frac{1}{T_{1 \mathrm{e}}}+\frac{1}{T_{1 \mathrm{p}}}
\end{array}\right.
$$

I.3. Le spin fictif et son évolution. - I.3.1. L'HAmiltonien D'Un ATOME. - Soient $E_{2}$ et $E_{4}$ les valeurs propres de l'énergie des états $|1,0\rangle$ et $|0,0\rangle$ respectivement. Elles sont telles que :

$$
E_{2}-E_{4}=h \omega_{0}
$$

où $\omega_{0}$ est la pulsation de la transition hyperfine.

En présence d'une induction magnétique dépendant du temps $B(t)$, parallèle au champ directeur, les éléments de matrice de perturbation sont [21] :

$$
V_{22}=V_{44}=0 ; \quad V_{24}=\mu_{\mathrm{B}} B(t) ; \quad V_{42}=V_{24}^{*}
$$

où $\mu_{B}$ est la valeur du magnéton de Bohr $\left(\mu_{B}>0\right)$.
L'hamiltonien $H$ d'un atome soumis à la perturbation cohérente est alors, en prenant pour origine des énergies la valeur $\frac{E_{2}+E_{4}}{2}$ :

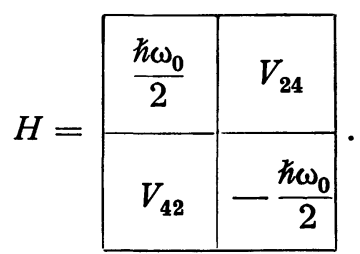

I.3.2. LE SPIN FICTIF. - I.3.2.1. Définition du spin fictif et de l'espace fictif. - Suivant Feynman [22] et Abragam [23], l'équation de Schrödinger décrivant l'évolution d'un système à deux niveaux est équivalente à l'équation du mouvement d'un spin fictif $\mathbf{r}$ soumis à une induction magnétique $\boldsymbol{\Omega} ; \boldsymbol{r}$ et $\boldsymbol{\Omega}$ sont définis par :

$$
\begin{array}{r}
\mathbf{r}=\operatorname{Tr}(\rho \sigma)\left\{\begin{array}{l}
r_{1}=\rho_{24}+\rho_{42} \\
r_{2}=i\left(\rho_{24}-\rho_{42}\right) \\
r_{3}=\rho_{22}-\rho_{44}
\end{array}\right. \\
\hbar \boldsymbol{\Omega}=\operatorname{Tr}(H \sigma)\left\{\begin{array}{l}
\hbar \Omega_{1}=V_{24}+V_{42} \\
\hbar \Omega_{2}=i\left(V_{24}-V_{42}\right) \\
\hbar \Omega_{3}=\hbar \omega_{0}
\end{array}\right.
\end{array}
$$

$\sigma$ est l'opérateur vectoriel de Pauli.

L'équation du mouvement du spin fictif, en l'absence de relaxation, est :

$$
\frac{\partial \mathbf{r}}{\partial t}=-\mathbf{r} \wedge \boldsymbol{\Omega}
$$

Dans le cas d'une transition $\Delta M=0$, l'espace à trois dimensions où les vecteurs $\boldsymbol{r}$ et $\boldsymbol{\Omega}$ évoluent est distinct de l'espace physique.

G'est un espace fictif dont l'axe 3 porte le champ directeur fictif (exprimé en unités de pulsation). Celui-ci est très grand devant le module du champ variable $\left(\Omega_{3} /\left|\Omega_{1}\right| \sim 10^{9}\right)$. En conséquence, l'effet BlochSiegert est négligeable. Nous ne considérons donc que la composante du champ variable fictif tournant dans le sens positif du plan 1,2 (par exemple). Ce plan est le plan de phase des grandeurs fonctions sinusoïdales du temps. Les composantes du champ tournant y sont :

$$
\begin{array}{r}
\Omega_{1}=b \cos (\omega t+\varphi) ; \quad \Omega_{2}=b \sin (\omega t+\varphi) \\
\omega \not \# \omega_{0} .
\end{array}
$$

L'amplitude du champ tournant, exprimée en unités de pulsation, est :

$$
b=\mu_{\mathrm{B}} B / \hbar
$$

où $B$ est l'amplitude de l'induction magnétique hyperfréquence appliquée aux atomes.

Le moment magnétique des atomes, induit par le champ variable, est :

$$
\mu=\mu_{B}\left(\rho_{24}+\rho_{42}\right)=\mu_{B} r_{1} .
$$


Nous appelons $r_{1}$ le moment magnétique réduit, $r_{2}$ est la grandeur conjuguée (au sens de la mécanique classique), $r_{3}$ est la différence de population entre les états $|1,0\rangle$ et $|0,0\rangle$, rapportée à un atome.

I.3.2.2. Évolution en présence de relaxation. - L'effet de la perturbation cohérente se superpose à celui de la relaxation : l'opérateur densité évolue peu - dans la représentation d'interaction - pendant un intervalle de temps égal au plus grand des temps de corrélation des différentes perturbations. Le temps de corrélation $\tau_{0}$ à considérer correspond à la relaxation par champ magnétique inhomogène. Il est de l'ordre de $10^{-4} \mathrm{~s}$, temps de vol moyen des atomes dans le ballon.

Gette dernière condition s'exprime avec nos notations, $b \tau_{\mathrm{c}} \ll 1$. Elle est toujours largement remplie.

En présence de relaxation, l'équation d'évolution du spin fictif devient donc, compte tenu des équations (5) et (9) :

$$
\frac{\partial \mathbf{r}}{\partial t}=-\mathbf{r} \wedge \boldsymbol{\Omega}-\frac{r_{1} \mathbf{k}_{1}+r_{2} \mathbf{k}_{2}}{T_{2}^{\prime \prime}}-\frac{r_{3} \mathbf{k}_{3}}{T_{1}^{\prime \prime}}
$$

où $\mathbf{k}_{1}, \mathbf{k}_{2}, \mathbf{k}_{3}$ sont les vecteurs de base orthonormés de l'espace fictif.

I.3.3. LE GONFINEMENT DANS LE BALLON. I.3.3.1. Moyenne sur l'instant d'arrivée. - Dans le ballon, la loi de répartition des temps de séjour est exponentielle, de la forme $\exp -\left(\frac{t-t_{0}}{T_{\mathrm{b}}}\right)$, où $t$ est l'instant d'observation, $t_{0}$ est l'instant d'arrivée, $T_{\mathrm{b}}$ est le temps de séjour moyen des atomes dans le ballon.

L'état global du système, à l'instant $t$, dépend de l'instant $t_{0}$ d'arrivée des atomes de deux façons différentes :

1) La contribution aux propriétés du système des atomes qui ont pénétré dans le ballon à l'instant $t_{0}$ doit être pondérée par leur probabilité de présence à l'instant $t$ d'observation;

2) Les atomes considérés dans le modèle à deux niveaux qui pénètrent dans le ballon à l'instant $t$ sont, en pratique, dans l'état pur $|1,0\rangle$ [24], [25]. Nous appelons $\rho(t, t)$ l'opérateur densité de ces atomes.

Le sous-ensemble $\left(t, t_{0}\right)$ des atomes qui sont arrivés à l'instant $t_{0}\left(t_{0}<t\right)$ a acquis un état mélange. Nous appelons $\rho\left(t, t_{0}\right)$ l'opérateur densité de ce sousensemble.

L'opérateur densité global $\rho(t)$ des atomes considérés ici est alors :

$$
\begin{gathered}
N(t) \rho(t)=\int_{t_{0}=0}^{t_{0}=t} \rho\left(t, t_{0}\right) I\left(t_{0}\right) \mathrm{d} t_{0} \exp -\left(\frac{t-t_{0}}{T_{\mathrm{b}}}\right) \\
N(t)=\int_{t_{0}=0}^{t_{0}=t} I\left(t_{0}\right) \mathrm{d} t_{0} \exp -\left(\frac{t-t_{0}}{T_{\mathrm{b}}}\right) \\
I\left(t_{0}\right)=0 \text { pour } t_{0} \leqslant 0 .
\end{gathered}
$$

L'opérateur $\rho(t)$ permet le calcul des grandeurs macroscopiques du système.
Le mouvement de $N(t) \rho(t)$ est déterminé par celui de $\rho\left(t, t_{0}\right)$ que nous avons rappelé aux paragraphes précédents. Par dérivation sous le signe somme, nous obtenons :

$$
\begin{array}{rl}
\frac{\mathrm{d}}{\mathrm{d} t}[N(t) \rho(t)]=\int_{t_{0}}=0 & I\left(t_{0}\right) \mathrm{d} t_{0} \mathrm{e}^{-\frac{t-t_{0}}{T_{\mathrm{b}}}} \frac{\partial}{\partial t} \rho\left(t, t_{0}\right) \\
& -\frac{N(t) \rho(t)}{T_{\mathrm{b}}}+I(t) \rho(t, t) .
\end{array}
$$

La méthode utilisée est analogue à celle introduite par Lamb dans sa théorie du laser [26], [27], où les états purs sont obtenus par un procédé différent.

Elle nous a permis de généraliser, au cas où le flux d'atomes $I(t)$ n'est pas constant, l'emploi du formalisme de l'opérateur densité pour décrire le comportement d'un maser à deux niveaux.

I.3.3.2. Moyenne sur le mouvement des atomes. - Le mouvement des atomes est aléatoire, dans une région où la phase du champ électromagnétique est constante et où son amplitude varie peu. Les atomes sont donc soumis à une perturbation cohérente, de fréquence bien déterminée, modulée faiblement en amplitude.

Ramsey [21] a montré qu'en conséquence :

- L'élargissement par effet Doppler de la raie de résonance du système atomique est négligeable;

- Le décalage en fréquence du sommet de la raie, dû à un léger défaut de symétrie du système, est négligeable;

- Tout se passe comme si les atomes étaient immobiles, soumis à une induction magnétique d'amplitude $B$, indépendante de la position, égale à la valeur moyenne sur le volume du ballon de la composante $B_{z}$ de l'amplitude de l'induction magnétique du mode $T E_{011}$.

Tout se passe donc comme si le champ magnétique réduit avait l'amplitude :

$$
b=\frac{\mu_{\mathrm{B}}\left\langle B_{z}\right\rangle_{\text {ballon }}}{\hbar} \text {. }
$$

I.3.4. L'ÉQUATION DE BLOGH DU SYSTÈME A DEUX NIVEAUX. - Nous introduisons l'ensemble des spins fictifs $\mathbf{R}$ tels que :

$$
\mathbf{R}=\int_{t_{0}=0}^{t_{0}=t} \mathbf{r}\left(t, t_{0}\right) I\left(t_{0}\right) \mathrm{e}^{-\frac{t-t_{0}}{T_{\mathrm{b}}}} \mathrm{d} t_{0}
$$

$R_{1}$ est le moment magnétique de l'ensemble des atomes, $R_{2}$ est la grandeur conjuguée, $R_{3}$ est la différence de population entre les états $|1,0\rangle$ et $|0,0\rangle$.

Compte tenu des équations (13) et (15), l'équation d'évolution de $\mathbf{R}$ est :

$$
\left\{\begin{array}{c}
\frac{\mathrm{d} \mathbf{R}}{\mathrm{d} t}=-\mathbf{R} \wedge \boldsymbol{\Omega}-\frac{R_{1} \mathbf{k}_{1}+R_{2} \mathbf{k}_{2}}{T_{2}}-\left[\frac{R_{3}}{T_{1}}-I(t) r_{0}\right] \mathbf{k}_{\mathbf{3}} \\
\frac{1}{T_{1}}=\frac{1}{T_{1 \mathrm{p}}}+\frac{1}{T_{1 \mathrm{e}}}+\frac{1}{T_{\mathrm{b}}} ; \quad \frac{1}{T_{2}}=\frac{1}{T_{2 \mathrm{p}}}+\frac{1}{T_{2 \mathrm{e}}}+\frac{1}{T_{\mathrm{b}}} \\
r_{0}=\operatorname{Tr}\left[\rho(t, t) \cdot \sigma_{3}\right] .
\end{array}\right.
$$


Les constantes de temps $T_{1}$ et $T_{2}$ dépendent de la densité d'atomes dans le ballon, par l'intermédiaire des constantes de l'échange de spin ( $T_{1 \mathrm{e}}$ et $T_{2 \mathrm{e}}$ sont inversement proportionnels à la densité d'atomes), et éventuellement, par l'intermédiaire de $T_{1 \mathrm{p}}$ et $T_{2 \mathrm{p}}$, s'il existe un effet de paroi dépendant de la densité [19].

Nous nous limiterons ici au cas où le flux d'atomes a une valeur constante, imposée depuis un intervalle de temps long devant $T_{\mathrm{b}}$. On a alors $N=I T_{\mathrm{b}}$, et en introduisant $R_{0}=N r_{0}$, on obtient :

$$
\frac{\mathrm{d} \mathbf{R}}{\mathrm{d} t}=-\mathbf{R} \wedge \boldsymbol{\Omega}-\frac{R_{1} \mathbf{k}_{1}+R_{2} \mathbf{k}_{2}}{T_{2}}-\frac{R_{3}-R_{0}\left(T_{1} / T_{\mathrm{b}}\right)}{T_{1}} \mathbf{k}_{3} .
$$

L'évolution du système atomique est donc décrite, dans l'espace figuratif défini plus haut, par une équation de Bloch.

Il est ainsi possible, en se plaçant dans l'espace fictif, de décrire classiquement les expériences effectuées sur le maser à hydrogène, en particulier la mesure du temps de relaxation $T_{2}$ par la méthode de l'impul$\operatorname{sion} \pi / 2$ [25].

I.3.5. Comportement DU SYSTÈme ATOMIQUE. I.3.5.1. Mouvement du moment magnétique. - En éliminant $R_{2}$ entre les projections, sur les axes 1 et 2 , de l'équation (19), en remarquant que les excitations $\Omega_{1}$ et $\Omega_{2}$ sont d'amplitude lentement variable, que $\omega$ est très voisin de $\omega_{0}$ et que $\omega_{0} T_{2}$ est très grand $\left(\omega_{0} T_{2} \simeq 2 \times 10^{9}\right)$, il vient :

$$
\ddot{R}_{1}+\frac{2}{T_{2}} \dot{R}_{1}+\omega_{0}^{2} R_{1}=2 \omega_{0} R_{3} \Omega_{1} .
$$

I.3.5.2. Mouvement de la différence de population entre les états $|1,0\rangle$ et $|0,0\rangle$. - En projetant l'équation (19) sur l'axe 3 de l'espace figuratif, et en faisant les mêmes remarques que précédemment, il vient :

$$
\dot{R}_{3}+\frac{R_{3}}{T_{1}}=-2\left(\overline{\frac{\Omega_{1}}{\omega_{0}} \dot{R}_{1}}\right)+\frac{R_{0}}{T_{\mathrm{b}}} .
$$

Le premier membre de cette équation est à variation lente, il est donc sensible à la valeur moyenne du second membre.

I.3.5.3. Conclusion. - Les équations couplées (20) et (21) décrivent le comportement du système atomique, excité par une perturbation cohérente, en présence de relaxation. La première équation représente les propriétés de résonance, à la pulsation $\omega_{0}$. Le couplage, par l'intermédiaire du terme $R_{3}$, traduit la saturation de la raie de résonance.

I.4. L'induction magnétique dans la cavité résonnante. - En pratique, le mode $\mathrm{TE}_{011}$ - dont la dégénérescence est levée par la présence du ballon de quartz - est bien séparé des modes voisins. G'est le seul mode qu'il y ait lieu de considérer.
Il est excité par l'aimantation du gaz d'hydrogène vibrant à une fréquence sensiblement égale à la fréquence propre du mode.

L'aimantation est uniforme sur le volume du ballon, puisque, en vertu du mouvement des atomes, l'induction magnétique variable peut y être considérée comme uniforme.

L'emploi des équations de Maxwell permet d'établir la dépendance temporelle de l'induction magnétique [28] qui s'écrit ici :

$$
\begin{gathered}
\ddot{\Omega}_{1}+\frac{2}{T_{\mathrm{c}}} \dot{\Omega}_{1}+\omega_{\mathrm{c}}^{2} \Omega_{1}=K_{1} \ddot{R}_{1} \\
T_{\mathrm{o}}=\frac{2 Q_{\mathrm{c}}}{\omega_{\mathrm{c}}} ; \quad K_{1}=\frac{\eta}{V_{\mathrm{o}}} \mu_{0} \frac{\mu_{\mathrm{B}}^{2}}{\hbar} ; \quad \eta=\frac{\left\langle B_{\mathrm{Z}}\right\rangle_{\text {ballon }}^{2}}{\left\langle B^{2}\right\rangle_{\text {cavite }}}
\end{gathered}
$$

où $T_{0}$ est la constante de temps de variation de l'amplitude du champ électromagnétique dans la cavité; $\omega_{\mathrm{e}}$ est la pulsation propre de la cavité; $Q_{\mathrm{e}}$ est son coefficient de surtension; $\eta$ est le coefficient de remplissage de la cavité par le milieu actif [15]; $V_{\mathrm{o}}$ est le volume de la cavité; $\mu_{0}$ est la perméabilité magnétique du vide ${ }^{(4)}$.

I.5. Equations aux amplitudes et équations aux phases. - Les équations (20), (21) et (22) déterminent en toute généralité le comportement du maser à hydrogène. Elles sont analogues à celles qui ont été établies, par une démarche différente, par Orayevskiy et Uspenskiy [10]. Elles s'appliquent à tout maser où l'approximation à deux niveaux est valable, et où la probabilité pour que les particules participant à la transition restent en présence du champ électromagnétique pendant l'intervalle de temps $\Delta t$ est donnée par une loi en : $\exp \left(-\Delta t / T_{\mathrm{b}}\right)$.

Nous cherchons des solutions de la forme :

$$
\Omega_{1}=b \cos (\omega t+\varphi) ; \quad R_{1}=m \sin (\omega t+\psi)
$$

les paramètres $b, m, \varphi, \psi$ sont des fonctions lentement variables du temps. Les conditions suivantes sont toujours très largement remplies :

$$
\frac{\dot{b}}{b}, \frac{\dot{m}}{m}, \frac{\dot{\varphi}}{\varphi}, \frac{\dot{\psi}}{\psi} \ll \omega_{0} .
$$

Les équations aux amplitudes sont alors :

$$
\begin{gathered}
\dot{m}+\frac{m}{T_{2}}=R_{3} b \cos (\varphi-\psi) \\
\dot{R}_{3}+\frac{R_{3}}{T_{1}}=-b m \cos (\varphi-\psi)+\frac{R_{0}}{T_{\mathrm{b}}} \\
\dot{b}+\frac{b}{T_{\mathrm{c}}}=\frac{1}{2} K_{1} m \omega \cos (\varphi-\psi)
\end{gathered}
$$

(4) Nous utilisons le système MKSA rationalisé. 
et les équations aux phases :

$$
\begin{gathered}
\dot{\psi}=\left(\omega_{0}-\omega\right)-R_{3} \frac{b}{m} \sin (\psi-\varphi) \\
\dot{\varphi}=\left(\omega_{\mathrm{c}}-\omega\right)-\frac{1}{2} K_{1} \frac{m}{b} \omega \sin (\varphi-\psi) .
\end{gathered}
$$

I.6. Un modèle radioélectrique pour l'étude du maser. - Les équations qui décrivent - en valeurs instantanées - le comportement du maser sont :

$$
\begin{gathered}
\ddot{R}_{1}+\frac{2}{T_{2}} \dot{R}_{1}+\omega_{0}^{2} R_{1}=2 R_{3} \Omega_{1} \omega_{0} \\
\dot{R}_{3}+\frac{R_{3}}{T_{1}}=-b m \cos (\varphi-\psi)+\frac{R_{0}}{T_{\mathrm{b}}} \\
\ddot{\Omega}_{1}+\frac{2}{T_{\mathrm{c}}} \dot{\Omega}_{1}+\omega_{\mathrm{c}}^{2} \Omega_{1}=-\omega^{2} K_{1} R_{1} .
\end{gathered}
$$

Elles suggèrent le modèle radioélectrique de la figure 3 comprenant :

1) Un amplificateur très sélectif représentant les propriétés de résonance du système atomique;

2) Un circuit de réaction peu sélectif;

3) Un contrôle de gain de l'amplificateur.
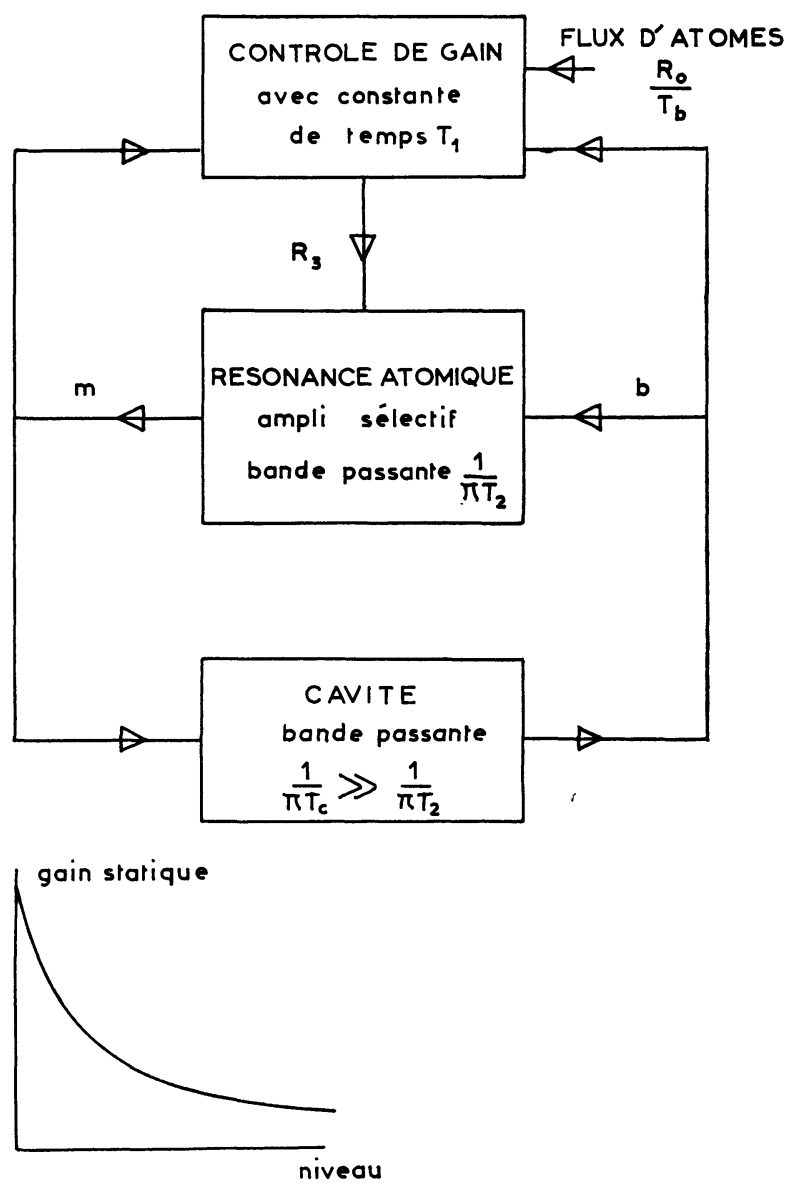

FIG. 3. - Modèle radioélectrique représentant le comportement du maser.
Le gain est commandé avec retard $\left(T_{1}\right)$ par la tension de sortie d'un détecteur de phase. Le gain est fonction décroissante du niveau d'oscillation, ce qui traduit la saturation de la transition atomique. Il peut être ajusté en réglant la tension continue $R_{0} / T_{\mathrm{b}}$ qui représente le flux d'atomes d'état $|1,0\rangle$.

La caractéristique essentielle de cet oscillateur est que le dispositif de limitation d'amplitude (du type " limiteur à filtre d'enveloppe ») agit avec une constante de temps $T_{1}$ qui est du même ordre de grandeur que la constante de temps $T_{2}$ de réponse du milieu actif aux variations d'amplitude. C'est ce fait qui explique que le comportement, au voisinage du régime stabilisé, du maser est différent de celui des oscillateurs classiquement étudiés [8].

En effet, le retour au niveau stabilisé de l'amplitude d'oscillation est exponentiel amorti pour ceux-ci. Nous verrons que, pour le maser à hydrogène, il peut être oscillatoire amorti.

I.7. Le régime stabilisé. - Le régime stabilisé est atteint lorsque les conditions expérimentales sont fixées depuis un intervalle de temps long devant $T_{1}$ et $T_{2}$. Les amplitudes et les phases sont alors des constantes.

Les équations précédemment établies permettent évidemment de retrouver les lois du régime stabilisé. Nous rappelons brièvement les résultats que nous utiliserons par la suite.

Le paramètre $b$ caractérise l'amplitude du champ électromagnétique dans la cavité. Au régime stabilisé, il est tel que :

$b_{\mathrm{s}}^{2}=K_{1} Q_{\mathrm{c}} \frac{R_{0}}{T_{\mathrm{b}}}-\frac{1}{T_{1} T_{2}}\left[1+4\left(\frac{\Delta f}{f_{0}}\right)^{2} Q_{\mathrm{c}}^{2}\right]$

où $\Delta f$ est l'écart entre la fréquence propre de la cavité et la fréquence $f_{0}$ de résonance du système atomique. A l'accord, lorsque toutes les conditions expérimentales sont fixées, sauf le coefficient de surtension de la cavité, le seuil d'oscillation est tel que :

$$
Q^{\prime} K_{1} \frac{R_{0}}{T_{\mathrm{b}}} T_{1} T_{2}=1
$$

La puissance $P_{\mathrm{a}}$ rayonnée par les atomes a pour expression, à l'accord de la cavité sur $f_{0}$ [7] :

$$
P_{\mathrm{a}}=\frac{1}{2} \hbar \omega_{0} \frac{R_{0}}{T_{\mathrm{b}}} \frac{T_{1} T_{2} b_{\mathrm{s}}^{2}}{1+T_{1} T_{2} b_{\mathrm{s}}^{2}} .
$$

La formule (31) montre que la puissance fournie aux circuits de mesure, proportionnelle à $b_{\mathrm{s}}^{2}$, est une fonction linéaire du coefficient de surtension et que la pente de la droite représentative est proportionnelle à $R_{0}$ et donc à la densité d'atomes dans le ballon [29].

I.8. Le régime transitoire. - I.8.1. LA TENDANGE VERS L'ÉQUILIBRE. - Lorsque le niveau d'oscillation est volontairement écarté de sa valeur stabilisée $b_{\mathrm{g}}$, par valeur inférieure par exemple, le gain du milieu actif est plus élevé qu'au niveau d'équilibre. Lorsque la cause perturbatrice est supprimée, le niveau va 
alors croître, et le contrôle de gain agissant avec retard (constante de temps $T_{1}$ ), l'amplitude d'oscillation peut dépasser, par valeur supérieure, sa valeur d'équilibre.

L'amplitude du signal maser peut donc effectuer des oscillations de pompage autour de sa valeur d'équilibre.

Nous avons précisé les conditions sous lesquelles le niveau d'oscillation serait instable. Celles-ci n'ont pas été atteintes expérimentalement [25].

I.8.2. LE RÉGIME TRANSITOIRE AU VOISINAGE DU RÉGIME STABILISÉ [30]. - Lorsque la fréquence propre de la cavité est voisine de la fréquence de la transition atomique, le déphasage $(\varphi-\psi)$ est petit, et une étude détaillée montre qu'il le reste pendant le transitoire, si l'oscillateur est faiblement écarté de son régime d'équilibre. Le facteur trigonométrique des équations aux amplitudes est alors très voisin de 1 et nous pouvons considérer, avec une excellente approximation, que les équations aux amplitudes sont découplées des équations aux phases.

Dans les conditions expérimentales où la vitesse d'évolution de l'amplitude pour le système atomique est beaucoup plus lente que la vitesse d'établissement du champ électromagnétique dans la cavité résonnante, les équations (25), (26), (27) permettent d'obtenir :

$$
\begin{aligned}
& \frac{\mathrm{d}}{\mathrm{d} t}\left(\frac{\dot{b}}{b}\right)+\frac{1}{T_{1}} \frac{\dot{b}}{b}+T_{\mathrm{c}} b \dot{b} \\
& +b^{2}-b_{\mathrm{s}}^{2}=0\left\{\begin{array}{l}
T_{\mathrm{c}} \ll T_{1}, T_{2} \\
T_{\mathrm{c}} \frac{\mathrm{d}}{\mathrm{d} t}\left(\frac{\ddot{b}}{b}\right) \ll \frac{\ddot{b}}{b} .
\end{array}\right.
\end{aligned}
$$

$\mathrm{Au}$ voisinage du régime stabilisé, nous posons :

$$
b=b_{\mathrm{s}}+\varepsilon \quad \varepsilon \ll b_{\mathrm{s}} .
$$

L'équation (34) se linéarise alors en :

$$
T_{1} \ddot{\varepsilon}+\dot{\varepsilon}\left(1+T_{1} T_{\mathrm{c}} b_{\mathrm{s}}^{2}\right)+2 T_{1} b_{\mathrm{s}}^{2} \varepsilon=0 .
$$

Nous avons opéré, dans l'étude du régime transitoire, dans des conditions telles que $T_{1} T_{\mathrm{c}} b_{\mathrm{s}}^{2} \leqslant 0,01$, ce qui, en pratique, est équivalent à $Q_{\mathrm{c}} \leqslant 4 \times 10^{5}$.

Le comportement au voisinage du régime stabilisé est alors décrit par :

$$
T_{1} \ddot{\varepsilon}+\dot{\varepsilon}+2 T_{1} b_{\mathrm{s}}^{2} \varepsilon=0 \quad T_{1} T_{\mathrm{c}} b_{\mathrm{s}}^{2} \leqslant 0,01 \text {. }
$$

I.8.2.1. Mouvement exponentiel amorti. - Lorsque le niveau d'oscillation est faible $\left(8 T_{1}^{2} b_{\mathrm{s}}^{2}<1\right)$, le retour au niveau stabilisé est exponentiel amorti.

A niveau d'oscillation très faible $\left(8 T_{1}^{2} b_{\mathrm{s}}^{2} \ll 1\right)$, les deux constantes de temps sont $T_{1}$ et $1 / 2 T_{1} b_{\mathrm{s}}^{2}>T_{1}$. Le mouvement est d'autant plus lent que le niveau est plus faible. Cette conclusion est conforme à l'observation.

I 8.2.2. Mouvement oscillatoire amorti. — Si le niveau d'oscillation est élevé, le retour au niveau stabilisé est oscillatoire amorti, de la forme :

$$
\varepsilon=A \mathrm{e}^{-t / 2 T_{1}} \cos \left(\omega_{1} t+\Phi\right) ; \quad 8 T_{1}^{2} b_{\mathrm{s}}^{2}>1
$$

avec :

$$
\omega_{1}^{2}+\left(1 / 4 T_{1}^{2}\right)=2 b_{\mathrm{s}}^{2}
$$

$A$ et $\Phi$ sont des constantes, déterminées par les conditions initiales.

Ce comportement a été prévu, dans le cas du maser à ammoniac, par Grasyuk et Orayevskiy [9], mais il n'avait pas pu être observé à notre connaissance.

Le calcul est fondé sur l'hypothèse que la probabilité de présence des particules dans le résonateur est fonction exponentielle décroissante du temps. Cette circonstance est caractéristique du maser à hydrogène ; mais l'hypothèse ne nous paraît pas justifiée lorsque la cavité résonnante est transparente au faisceau de particules.

I.8.3. PRINGIPE DE LA MESURE DU TEMPS DE RELAXATION $T_{1}$ ET DE LA FRÉQUENGE DE NUTATION $b_{s}$. L'amplitude du mouvement oscillatoire amorti décroît avec la constante de temps $2 T_{1}$ qu'il est ainsi possible de déterminer [30]. La mesure de la pseudo-pulsation $\omega_{1}$ permet d'atteindre directement $b_{s}$, amplitude réduite du champ électromagnétique dans la cavité (fréquence de nutation).

La mesure de $T_{1}$ est commode lorsque le signal enregistré présente un grand nombre de maxima, c'est-à-dire lorsque la pseudo-pulsation est grande et le niveau d'oscillation élevé. C'est pourquoi nous associons à la cavité du maser une boucle de réaction comprenant un amplificateur paramétrique et un déphaseur. Nous réglons ainsi le coefficient de surtension apparent de la cavité et donc le niveau d'oscillation conformément à la relation (31). La figure 4 montre l'un des enregistrements obtenus.

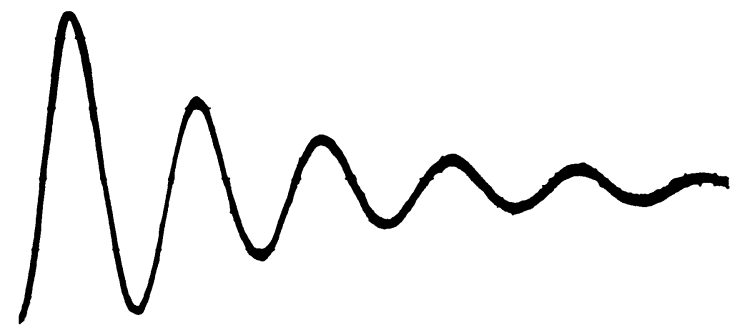

FIG. 4. - Retour oscillatoire amorti du niveau d'oscillation vers sa valeur d'équilibre. Pression source : $1,5 \times 10^{-1}$ torr $-Q_{\mathrm{c}}=350000$. La durée de l'enregistrement est de $1 \mathrm{~s}$.

I 8.4. ÉGART A LA LINÉARITÉ. - La non-linéarité de l'équation (39) limite l'amplitude à donner à la perturbation. Afin de préciser ce point, nous développons cette équation, au voisinage du régime stabilisé, au second ordre en $\varepsilon / b_{\mathrm{s}}$ :

$$
\frac{\ddot{\varepsilon}}{b_{\mathrm{s}}}+\frac{1}{T_{1}} \frac{\dot{\varepsilon}}{b_{\mathrm{s}}}-\left(\frac{\dot{\varepsilon}}{b_{\mathrm{s}}}\right)^{2}+2 \frac{\varepsilon}{b_{\mathrm{s}}}\left(1+\frac{3}{2} \frac{\varepsilon}{b_{\mathrm{s}}}\right) b_{\mathrm{s}}^{2}=0 .
$$

Cherchons une solution de la forme :

$\varepsilon / b_{\mathrm{s}}=A \mathrm{e}^{-t / 2 T_{1}} \cos \left(\omega_{1} t+\Phi\right)+x=\left(\varepsilon / b_{\mathrm{s}}\right)_{0}+x$. 
En première approximation, $x$ est solution de :

$$
\ddot{x}+\frac{1}{T_{1}} \dot{x}+2 b_{\mathrm{s}}^{2} x=\left(\frac{\dot{\varepsilon}}{b_{\mathrm{s}}}\right)_{0}^{2}-3 b_{\mathrm{s}}^{2}\left(\frac{\varepsilon}{b_{\mathrm{s}}}\right)_{0}^{2} .
$$

La solution $x$ est somme de trois termes qui sont :

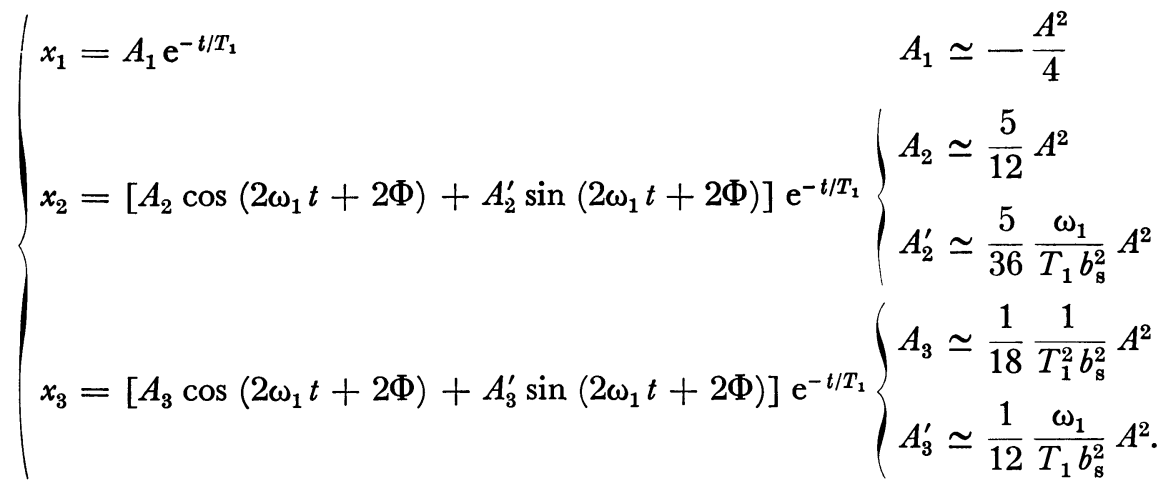

Il se superpose au mouvement en

$$
\mathrm{e}^{-t / 2 T_{1}} \cos \left(\omega_{1} t+\Phi\right)
$$

un mouvement exponentiel et un mouvement oscillatoire de pulsation $2 \omega_{1}$ qui s'amortissent plus vite que le terme principal.

En pratique, nous avons opéré dans des conditions telles que l'amplitude maximale du régime transitoire soit de $10 \%$ du niveau stabilisé. L'effet de la nonlinéarité est alors faible. La précision de la mesure de $T_{1}$ est, dans l'état actuel de notre montage de mesure, limitée par le bruit de fond du récepteur plutôt que par la non-linéarité. Cette précision est de $\pm 5 \%$.

\section{CONDITIONS DE VALIDITE DE L'APPROXIMATION A DEUX NIVEAUX}

Nous étudions plus particulièrement l'influence d'un champ magnétique inhomogène provoquant des transitions entre les sous-niveaux Zeeman $F=1$ et nous précisons les conditions - peu restrictives - sous lesquelles la mesure de $T_{1}$ conserve un sens [31].

II.1. Développement de l'opérateur densité sur une base d'opérateurs orthonormés. - La matrice densité a une structure en échelon particulièrement simple (fig. 1).

Si l'on envisage l'espace de Hilbert à 16 dimensions, dont les vecteurs de base sont les produits d'états propres $|F, M\rangle\left\langle F^{\prime}, M^{\prime}\right|$, le vecteur représentant la matrice densité a des composantes non nulles :

- soit dans le sous-espace à quatre dimensions des états $|1,0\rangle$ et $|0,0\rangle$;

- soit dans le sous-espace à une dimension de l'état $|1,1\rangle$;

- soit dans le sous-espace à une dimensions de l'état $|1,-1\rangle$.
En conséquence, six opérateurs orthonormés, linéairement indépendants, constituent un ensemble complet d'opérateurs de base [12].

Nous considérons ici (fig. 5) :

- d'une part, les opérateurs $\sigma_{1}, \sigma_{2}, \sigma_{3}, I_{\mathrm{A}}$ définis dans le sous-espace des états $|1,0\rangle$ et $|0,0\rangle$. Ce sont ceux que nous avons considérés dans l'étude de l'approximation à deux niveaux;

- d'autre part, les opérateurs $F_{\mathrm{Z}}$ et $I_{\mathrm{B}}$ appartenant aux sous-espaces de l'état $|1,1\rangle$ et de l'état $|1,-1\rangle$.

L'opérateur densité se développe alors selon :

$\rho=r_{1} \sigma_{1}+r_{2} \sigma_{2}+r_{3} \sigma_{3}+r_{4}^{\prime} I_{\mathrm{A}}+r_{5} F_{\mathrm{Z}}+r_{6} I_{\mathrm{B}}$.

Les composantes $r_{\mathrm{i}}$ sont définies par :

$r_{1}=\operatorname{Tr}\left(\rho \sigma_{1}\right) ; \quad r_{2}=\operatorname{Tr}\left(\rho \sigma_{2}\right) ; \quad r_{3}=\operatorname{Tr}\left(\rho \sigma_{3}\right)$

$r_{4}^{\prime}=\frac{1}{2} \operatorname{Tr}\left(\rho I_{\mathrm{A}}\right) ; \quad r_{5}=\operatorname{Tr}\left(\rho F_{\mathrm{Z}}\right) ; \quad r_{6}=\frac{1}{2} \operatorname{Tr}\left(\rho I_{\mathrm{B}}\right)$.

Les grandeurs physiques correspondant à ces composantes sont, respectivement :

- le moment magnétique induit;

- le moment magnétique, conjugué au précédent, au sens de la mécanique classique;

- la différence de population entre les états $|1,0\rangle$ et $|0,0\rangle$, rapportée à un atome;

- la population totale des niveaux $|1,0\rangle$ et $|0,0\rangle$, rapportée à un atome;

- la polarisation atomique;

- la population totale des niveaux $|1,1\rangle$ et $|1,-1\rangle$, rapportée à un atome. 


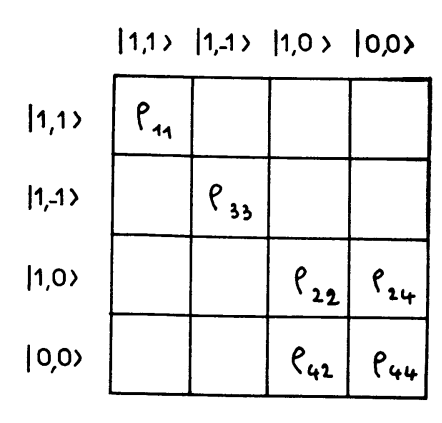

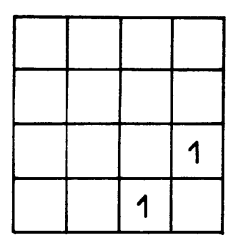

$\sigma_{1}$

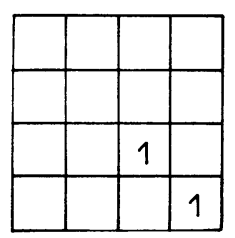

$I_{A}$

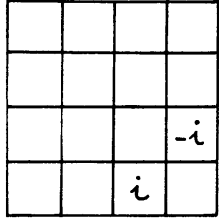

$\sigma_{2}$

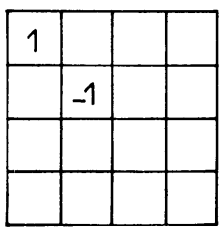

$F_{z}$

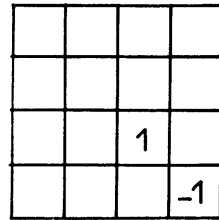

$\sigma_{3}$

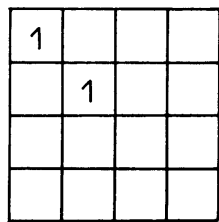

$I_{B}$
FIG. 5. - Opérateur densité

et opérateurs orthonormés dans la base $|F, M\rangle$.

Afin d'alléger l'écriture des relations suivantes, nous remplaçons $r_{4}^{\prime}$ par :

$$
r_{4}=1-4 r_{4}^{\prime}=1-2\left(\rho_{22}+\rho_{44}\right) .
$$

D'autre part, en conséquence de la relation $\operatorname{Tr} \rho=1$, $r_{6}$ est déterminé lorsque $r_{4}$ est connu.

II.2. Evolution des composantes de l'opérateur $\rho\left(t, t_{0}\right)$. - Comme au chapitre précédent, nous envisageons d'abord l'évolution de la matrice densité $\rho\left(t, t_{0}\right)$ du sous-groupe des atomes qui ont pénétré dans le ballon à l'instant $t_{0}$ (à $\mathrm{d} t_{0}$ près).

II.2.1. INFLUENGE DE LA RELAXATION PAR ÉGHANGE DE SPIN. - L'évolution de l'opérateur densité d'atomes d'hydrogène a été considérée en détail par Balling, Hanson et Pipkin [16] dont nous utilisons les résultats.

Nous omettons, ici, tous les termes correspondant à des effets inobservables dans les circonstances actuelles [25] (en particulier : termes liés à l'indiscernabilité des atomes d'hydrogène).

Nous ne tenons pas compte non plus du léger décalage de fréquence résultant de l'échange d'électron. Son effet sur le niveau d'oscillation du maser est parfaitement négligeable.

Avec nos notations, l'évolution de $\rho\left(t, t_{0}\right)$ par échange de spin s'écrit alors :

$$
\left\{\begin{array}{l}
\frac{\partial}{\partial t} r_{1}\left(t, t_{0}\right)=-\frac{1}{T_{2 \mathrm{e}}} r_{1}\left(t, t_{0}\right) \\
\frac{\partial}{\partial t} r_{2}\left(t, t_{0}\right)=-\frac{1}{T_{2 \mathrm{e}}} r_{\mathbf{2}}\left(t, t_{0}\right) \\
\frac{\partial}{\partial t} r_{3}\left(t, t_{0}\right)=-\frac{1}{T_{1 \mathrm{e}}} r_{3}\left(t, t_{0}\right) \\
\frac{\partial}{\partial t} r_{4}\left(t, t_{0}\right)=-\frac{1}{T_{1 \mathrm{e}}} r_{4}\left(t, t_{0}\right)+\frac{1}{T_{1 \mathrm{e}}} r_{5}\left(t, t_{0}\right) r_{5}(t) \\
\frac{\partial}{\partial t} r_{5}\left(t, t_{0}\right)=0 .
\end{array}\right.
$$

II.2.2. INFLUence De LA RelaXation SUR LA PAROI. - Les équations les plus simples qui rendent compte de l'expérience sont [18] :

$$
\begin{aligned}
& \frac{\partial}{\partial t} r_{\mathrm{i}}\left(t, t_{0}\right)=-\frac{1}{T_{2 \mathrm{p}}} r_{\mathrm{i}}\left(t, t_{0}\right) \quad \text { pour } \quad i=1,2 \\
& \frac{\partial}{\partial t} r_{\mathrm{i}}\left(t, t_{0}\right)=-\frac{1}{T_{1 \mathrm{p}}} r_{\mathrm{i}}\left(t, t_{0}\right) \quad \text { pour } \quad i=3,4,5 \text {. }
\end{aligned}
$$

II.2.3. INFLUENGE D'UN GHAMP MAGNÉTIQUE INHOMOGÈNE. - Un champ magnétique inhomogène longitudinal n'a pratiquement pas d'influence à cause de la faible variation de la fréquence de la transition $\Delta M=0$ avec le champ magnétique (dépendance au second ordre). La raie de résonance reste homogène [21].

Sous l'effet d'un champ magnétique inhomogène transversal, les atomes sont soumis, dans leur mouvement, à un champ magnétique aléatoire, perpendiculaire au champ directeur. Le spectre de Fourier de la perturbation a une étendue de l'ordre de $1 / \tau_{0}$ où $\tau_{0}$ est le temps de vol moyen des atomes dans le ballon $\left(1 / \tau_{0} \sim 10 \mathrm{kHz}\right)$. Cette perturbation provoque des transitions entre les niveaux $F=1$ si le champ directeur a une valeur telle que l'écart $\omega_{Z}$ entre ces sous-niveaux Zeeman, exprimé en unité de pulsation, satisfait à l'inégalité $\omega_{Z} \tau_{0}<1$.

L'influence d'un champ magnétique inhomogène transversal est donc d'autant plus petite que le champ directeur a une valeur plus élevée.

La variation de la matrice densité, sous l'influence de cette perturbation, a été exprimée par Crampton [18], ainsi que par Vanier et Vessot [32]. Avec nos notations, elle s'écrit :

$$
\left\{\begin{array}{l}
\frac{\partial}{\partial t} r_{1}\left(t, t_{0}\right)=-\frac{1}{T_{\mathrm{m}}} r_{1}\left(t, t_{0}\right) \\
\frac{\partial}{\partial t} r_{2}\left(t, t_{0}\right)=-\frac{1}{T_{\mathrm{m}}} r_{2}\left(t, t_{0}\right) \\
\frac{\partial}{\partial t} r_{3}\left(t, t_{0}\right)=-\frac{1}{T_{\mathrm{m}}} r_{3}\left(t, t_{0}\right)+\frac{1}{T_{\mathrm{m}}} r_{4}\left(t, t_{0}\right) \\
\frac{\partial}{\partial t} r_{4}\left(t, t_{0}\right)=-\frac{2}{T_{\mathrm{m}}} r_{4}\left(t, t_{0}\right)+\frac{2}{T_{\mathrm{m}}} r_{3}\left(t, t_{0}\right) \\
\frac{\partial}{\partial t} r_{5}\left(t, t_{0}\right)=-\frac{1}{T_{\mathrm{m}}} r_{5}\left(t, t_{0}\right) .
\end{array}\right.
$$


La fonction d'autocorrélation de la perturbation est mal connue [21]. On admet, en général, que $T_{\mathrm{m}}$ satisfait à une relation du type :

$\frac{1}{T_{\mathrm{m}}}=\left(\frac{\gamma}{2}\right)^{2}\left\langle B_{\mathrm{t}}^{2}\right\rangle \frac{\tau_{\mathrm{o}}}{1+\left(\omega_{Z} \tau_{0}\right)^{n}}$ avec $n \geqslant 2$

où $\gamma$ est le rapport gyromagnétique de l'électron libre,

$B_{\mathrm{t}}$ est une composante transversale du champ magnétique inhomogène.

II.3. Evolution des composantes de l'opérateur densité global $\rho(t)$. - II.3.1. DÉFINITION DE L'OPÉRATEUR DENSITÉ GLOBAL ET DE SES COMPOSANTES. Le mouvement de l'opérateur densité global $\rho(t)$, introduit comme précédemment, est déterminé par celui des sous-ensembles $\left(t, t_{0}\right)$, par la composition du jet atomique et par la constante de temps de pompage du balton (formule (15)).

Les projections de l'opérateur $\rho(t)$ sur la base orthonormée définie plus haut sont :

$$
\begin{array}{r}
R_{\mathrm{i}}=N(t) r_{\mathrm{i}}(t)=\int_{t_{0}=0}^{t_{0}=t} I\left(t_{0}\right) r_{\mathrm{i}}\left(t, t_{0}\right) \mathrm{e}^{-\frac{t-t_{0}}{\mathrm{~T}_{\mathrm{b}}}} \mathrm{d} t_{0} \\
\text { pour } \quad i=1,2,3,4,5 .
\end{array}
$$

II.3.2. SUPERPOSITION DES DIFFÉRENTES GAUSES D'ÉVolution. - Comme précédemment, les effets des différentes perturbations, cohérentes ou aléatoires, se superposent.

En nous limitant ici au cas où le flux d'atomes et la composition du jet sont fixes, il vient :

$$
\left\{\begin{array}{l}
\frac{\mathrm{d}}{\mathrm{d} t} R_{1}=-\frac{1}{T_{2}} R_{1}-\omega_{0} R_{2}+\Omega_{2} R_{3} \\
\frac{\mathrm{d}}{\mathrm{d} t} R_{2}=\omega_{0} R_{1}-\frac{1}{T_{2}} R_{2}-\Omega_{1} R_{3} \\
\frac{\mathrm{d}}{\mathrm{d} t} R_{3}=\frac{R_{0}}{T_{\mathrm{b}}}-\Omega_{2} R_{1}+\Omega_{1} R_{2}-\frac{1}{T_{1}} R_{3}+\frac{1}{T_{\mathrm{m}}} R_{4} \\
\frac{\mathrm{d}}{\mathrm{d} t} R_{4}=\frac{R_{0}^{\prime}}{T_{\mathrm{b}}}+\frac{2}{T_{\mathrm{m}}} R_{3}-\frac{1}{T_{1}} R_{4}-\frac{1}{T_{\mathrm{m}}} R_{4}+\frac{1}{T_{1 \mathrm{e}}} \frac{R_{5}^{2}}{N_{\mathrm{tot}}} \\
\frac{\mathrm{d}}{\mathrm{d} t} R_{5}=\frac{R_{0}^{\prime \prime}}{T_{\mathrm{b}}}-\left(\frac{1}{T_{1 \mathrm{p}}}+\frac{1}{T_{\mathrm{m}}}+\frac{1}{T_{\mathrm{b}}}\right) R_{5}
\end{array}\right.
$$

avec :

$$
\left\{\begin{array}{l}
R_{0}=N_{\text {tot }} \operatorname{Tr}\left[\rho(t, t) \sigma_{3}\right] \\
R_{0}^{\prime}=N_{\text {tot }}\left\{1-\operatorname{Tr}\left[\rho(t, t) I_{\mathrm{A}}\right]\right\} \\
R_{0}^{\prime \prime}=N_{\text {tot }} \operatorname{Tr}\left[\rho(t, t) F_{Z}\right] \\
\frac{1}{T_{1}}=\frac{1}{T_{1 \mathrm{p}}}+\frac{1}{T_{1 \theta}}+\frac{1}{T_{\mathrm{m}}}+\frac{1}{T_{\mathrm{b}}} \\
\frac{1}{T_{2}}=\frac{1}{T_{2 \mathrm{p}}}+\frac{1}{T_{2 \theta}}+\frac{1}{T_{\mathrm{m}}}+\frac{1}{T_{\mathrm{b}}}
\end{array}\right.
$$

$N_{\text {tot }}$ est le nombre total d'atomes dans le ballon.
II.3.3. Modification des ÉQuations de Blogh. Dans l'espace fictif défini au paragraphe I.3.2.1, l'équation d'évolution des composantes $R_{1}, R_{2}, R_{3}$ du spin fictif est :

$$
\begin{aligned}
\frac{\mathrm{d} \mathbf{R}}{\mathrm{d} t}=-\mathbf{R} \wedge \boldsymbol{\Omega} & -\frac{R_{1} \mathbf{k}_{1}+R_{2} \mathbf{k}_{2}}{T_{2}} \\
& -\frac{R_{3}-R_{0}\left(T_{1} / T_{\mathrm{b}}\right)}{T_{1}} \mathbf{k}_{3}+\frac{1}{T_{\mathrm{m}}} R_{4} \mathbf{k}_{3} .
\end{aligned}
$$

Les équations de Bloch (19) sont modifiées par l'introduction du terme en $\frac{1}{T_{\mathrm{m}}} R_{4}$ qui traduit le couplage des spins fictifs à la polarisation atomique des états $|1,1\rangle$ et $|1,-1\rangle$. Le degré de couplage dépend du degré d'inhomogénie du champ magnétique statique. Le couplage est nul lorsque ce champ peut être considéré comme uniforme, ainsi qu'il a été supposé dans la première partie.

II.4. Equations aux phases et aux amplitudes. En projection sur le plan 1-2 de l'espace fictif, les équations de Bloch ont la même forme que dans l'approximation à deux niveaux. Il en résulte que les équations aux phases (28) et (29) sont inchangées, ainsi que les équations aux amplitudes (25) et (27).

Seule l'équation en $R_{3}$ est modifiée par le couplage par champ magnétique inhomogène. Elle devient :

$$
\begin{gathered}
T_{1} \ddot{R}_{3}+\left(2+\frac{1}{\delta}\right) \dot{R}_{3}+\left(1+\frac{1}{\delta}-\frac{2}{\delta^{2}}\right) \frac{R_{3}}{T_{1}} \\
=-T_{1} \frac{\mathrm{d}}{\mathrm{d} t}[b m \cos (\varphi-\psi)] \\
-\left(1+\frac{1}{\delta}\right) b m \cos (\varphi-\psi) \\
+\frac{1}{\delta} \frac{R_{5}^{2}}{N_{\text {tot }} T_{1 \mathrm{e}}}+\left(1+\frac{1}{\delta}\right) \frac{R_{0}}{T_{\mathrm{b}}}+\frac{1}{\delta} \frac{R_{0}^{\prime}}{T_{\mathrm{b}}} \\
\text { avec } \\
\delta=T_{m} / T_{1} .
\end{gathered}
$$

II .5. Le régime stabilisé. - L'amplitude réduite du champ électromagnétique dans la cavité est :

$$
\begin{aligned}
b_{\mathrm{s}}^{2}=K_{1} Q_{\mathrm{c}} \frac{R_{0}}{T_{\mathrm{b}}}-\frac{1+\delta-2 / \delta}{T_{1} T_{2}(1+\delta)} \\
+K_{1} Q_{\mathrm{c}} \frac{\left(R_{\mathrm{b}}^{2}\right)_{\mathrm{s}}}{N_{\text {tot }}} \frac{1}{T_{1 \mathrm{e}}(1+\delta)}+\frac{K_{1} Q_{\mathrm{c}}}{1+\delta} \frac{R_{0}^{\prime}}{T_{\mathrm{b}}} .
\end{aligned}
$$

Le paramètre $K_{1}$ est défini par la relation (22).

II.6. Equation du régime transitoire. - Dans l'hypothèse où la sélectivité de la cavité est faible devant celle du système atomique, et où la fréquence propre de la cavité est égale à celle de la transition 
atomique, l'équation de l'amplitude du champ électromagnétique, $b$, est :

$$
\begin{aligned}
T_{1} \frac{\mathrm{d}^{2}}{\mathrm{~d} t^{2}}\left(\frac{\dot{b}}{b}\right)+\left(2+\frac{1}{\delta}\right) & \frac{\mathrm{d}}{\mathrm{d} t}\left(\frac{\dot{b}}{b}\right) \\
+ & \frac{1}{T_{1}}\left(1+\frac{1}{\delta}-\frac{2}{\delta^{2}}\right) \frac{\dot{b}}{b}+T_{1} \frac{\mathrm{d}}{\mathrm{d} t}\left(b^{2}\right) \\
+\left(1+\frac{1}{\delta}\right)\left(b^{2}-b_{\mathrm{s}}^{2}\right) & =\frac{1}{\delta} \frac{1}{T_{1 \mathrm{e}}} K_{1} Q_{\mathrm{c}} \frac{R_{5}^{2}(t)-\left(R_{5}^{2}\right)_{s}}{N_{\mathrm{tot}}} .
\end{aligned}
$$

Le second membre n'est pas nul lorsque la perturbation excitant le régime transitoire est une modification du champ magnétique inhomogène, se traduisant par une variation brusque de $T_{\mathrm{m}}$.

Au voisinage du régime stabilisé, l'équation précédente se linéarise en :

$$
\left\{\begin{array}{c}
T_{1} \dddot{\varepsilon}+\left(2+\frac{1}{\delta}\right) \ddot{\varepsilon}+\left(1+\frac{1}{\delta}-\frac{2}{\delta^{2}}\right) \frac{1}{T_{1}} \dot{\varepsilon}+2 T_{1} b_{\mathrm{s}}^{2} \dot{\varepsilon} \\
\quad+\left(1+\frac{1}{\delta}\right) 2 b_{\mathrm{s}}^{2} \varepsilon=\frac{K_{1} Q_{\mathrm{c}}}{\delta T_{1 \mathrm{e}}} b_{\mathrm{s}} \frac{R_{5}^{2}(t)-\left(R_{5}^{2}\right)_{s}}{N_{\text {tot }}} \\
b=b_{\mathrm{s}}+\varepsilon .
\end{array}\right.
$$

II.7. Constante de temps du mouvement exponentiel amorti. - Lorsque le niveau d'oscillation est suffisant pour que le retour au régime stabilisé soit oscillatoire amorti, les solutions de l'équation caractéristique de l'équation différentielle (59) sont :

$$
\begin{aligned}
& S_{1}=-\frac{1-A}{2 T_{1}} \\
& \pm i \frac{\left[-(1-A)^{2}+8 T_{1}^{2} b_{\mathrm{s}}^{2}\left(1-\frac{A}{1+1 / \delta+A}\right)\right]^{1 / 2}}{2 T_{1}} \\
& S_{3}=-\frac{1}{T_{1}}\left(1+\frac{1}{\delta}+A\right) .
\end{aligned}
$$

Le paramètre $A$ est solution de :

$$
\left(1+\frac{1}{\delta}+A\right)\left(-\frac{2}{\delta^{2}}+\frac{A}{\delta}+A^{2}\right)+2 T_{1}^{2} b_{\mathrm{s}}^{2} A=0 \text {. }
$$

Son développement en puissance de $1 / \delta$ est :

$$
A=\frac{1}{T_{1}^{2} b_{\mathrm{s}}^{2}}\left[\frac{1}{\delta^{2}}+\frac{1}{2}\left(2-\frac{1}{T_{1}^{2} b_{\mathrm{s}}^{2}}\right) \frac{1}{\delta^{3}}+\ldots\right] \text {. }
$$

Portons notre attention sur la solution (60). La pseudo-pulsation $\omega_{1}^{\prime}$ est telle que :

$$
\omega_{1}^{\prime 2}+(1-A)^{2} \frac{1}{4 T_{1}^{2}}=2 b_{\mathrm{s}}^{2}\left(1-\frac{A}{1+\frac{1}{\delta}+A}\right)
$$

L'enveloppe du mouvement décroît avec la constante de temps :

$$
2 \tau_{1}=2 T_{1} /(1-A) .
$$

Il est donc possible de mesurer le temps de relaxation $T_{1}$ en présence de champ magnétique inhomogène. L'erreur systématique résultant alors de la méthode de mesure est du second ordre en $1 / \delta=T_{1} / T_{\mathrm{m}}$. L'erreur est d'autant plus petite que le niveau d'oscillation est plus élevé.

Nous avons vérifié expérimentalement ces conclusions en produisant un champ magnétique inhomogène permettant de faire varier $1 / \delta$ de 0 à 0,5 environ [31]

Dans la pratique de la mesure de $T_{1}$, où les seules inhomogénies du champ magnétique sont résiduelles, il est toujours possible de s'affranchir de l'erreur systématique en opérant en présence d'un champ magnétique directeur de valeur suffisamment élevée (de l'ordre de 25 milligauss).

II.8. Constante d'intégration. Amplitude des mouvements parasites. - Le calcul des constantes d'intégration montre que, selon les circonstances expérimentales, la phase $\Phi$ de la solution en $\mathrm{e}^{-t / 2 T_{1}} \cos \left(\omega_{1} t+\Phi\right)$ peut varier entre 0 et $\pi / 2$, ce que nous avons observé.

$\mathrm{Au}$ mouvement oscillatoire amorti se superposent les mouvements exponentiels amortis correspondant, d'une part, à la solution en $\mathrm{e}^{s_{3} t}$ de l'équation (59) et à la solution de l'équation avec second membre, d'autre part. Nous avons observé ces solutions parasites lorsque le couplage des sous-niveaux Zeeman est fort.

Conclusion. - La vérification expérimentale des conclusions théoriques concernant le comportement dynamique de l'oscillateur et l'emploi d'une boucle

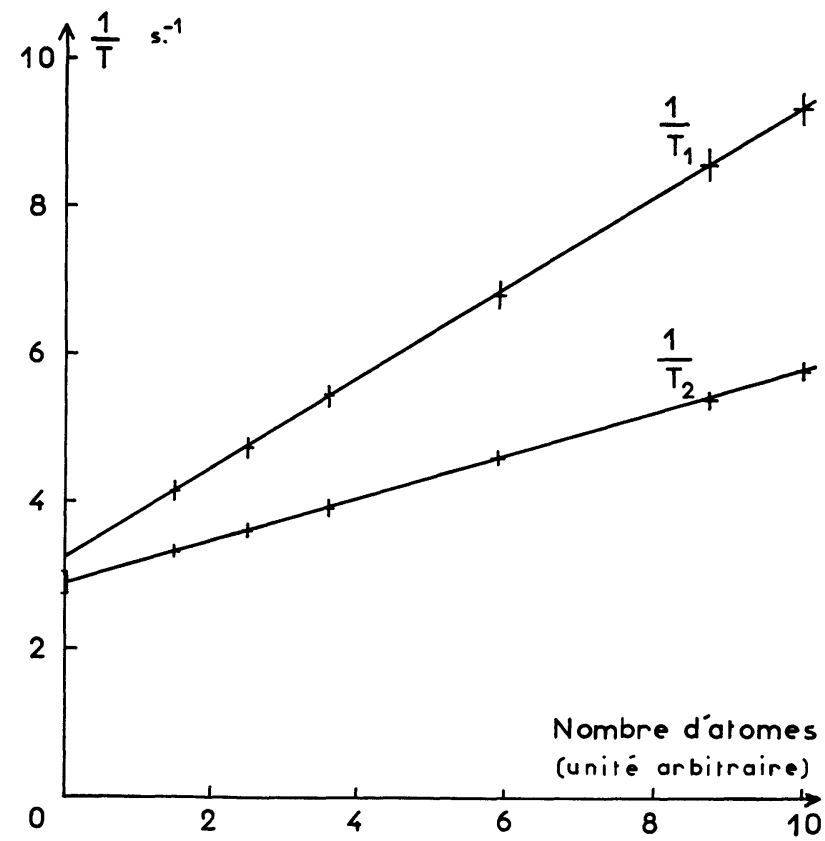

FIg. 6. - Variation des temps de relaxation $T_{1}$ et $T_{2}$ en fonction de la densité d'atomes. 
de réaction a été effectuée sur nos deux masers à hydrogène [33], [34], dont nous avons étudié en détail le séparateur magnétique hexapolaire [24], [25], [35], [36], [37], [38].

La figure 6 montre, dans les conditions d'oscillation, la variation des temps de relaxation $T_{1}$ et $T_{2}$ en fonction de la densité d'atomes dans le ballon. La constante de temps $T_{1}$ est mesurée en enregistrant le comportement dynamique du maser. La mesure de $T_{2}$ est classique [21]. L'axe des abscisses est gradué en valeurs relatives du flux d'atomes pénétrant dans le ballon. Ces valeurs sont obtenues en mesurant le niveau d'oscillation en fonction du coefficient de surtension de la cavité résonnante ( $(\mathrm{I} .7$ ).

Par trois méthodes de mesure différentes [29], nous avons atteint la valeur absolue du flux d'atomes pénétrant dans le ballon.
Nous en avons déduit la valeur de la section efficace d'échange de spin $\sigma$ entre atomes d'hydrogène :

$$
\sigma=(2,57 \pm 0,50) \times 10^{-15} \mathrm{~cm}^{2} .
$$

Ces méthodes nous ont permis de mesurer également la valeur du paramètre $q$ [7] qui caractérise l'effet de l'échange de spin sur le niveau d'oscillation du maser. Lorsque le coefficient de surtension de la cavité résonnante vaut 50700 , la valeur de $q$ est, pour notre maser de structure verticale :

$$
q=0,053 \pm 0,005 \text {. }
$$

Cette valeur permet d'obtenir un niveau d'oscillation élevé.

Tous les résultats de mesure, qui feront l'objet d'une publication séparée, forment un ensemble cohérent confirmant la validité de prévisions théoriques.

Manuscrit reçu le 4 juillet 1967.

\section{BIBLIOGRAPHIE}

[1] GOLDENBERG (H. M.), KLEPPNER (D.), RAMSEY (N. F.), Phys. Rev. Lett., 1960, 5, 361.

[2] Peters (H. E.), Holloway (J.), Bagley (A. S.), Cutrer (L. S.), Appl. Physics Letters, 1965, 6, 2, 34.

Peteres (H. E.), KarTaschoff (P.), Appl. Physics Letters, 1965, 6, 2, 35.

[3] Vessot (R. F. C.), Peters (H. E.), IRE Trans. Instr., 1962, 11, 183.

[4] Menound (C.), Racine (J.), Helv. Physica Acta, $1962,35,562$.

[5] Basov (N. G.), Strakhovskiy (G. M.), NIKITIN (A. I.), Nikitina (T. F.), TATARENKOV (V. M.), USPENSKIY (A. V.), Radio Engin. Electronic Physics, 1965, 10, 1551.

[6] Crampton (S. B.), Robinson (H. G.), KLEPPNER (D.), RAmsey (N. F.), Phys. Rev., 1966, 141, 55.

[7] KieppNer (D.), Berg (H. C.), CRAMPtoN (S. B.), RAMSey (N. F.), Vessot (R. F. C.), Peters (H. E.), VANier (J.), Phys. Rev., 1965, 138, A 972.

[8] BLAQUiÈre: (A.), Ann. Radioélectricité, 1953, 8, 36 et 153.

[9] Grasyuk (A. Z.), Orayevskiy (A. N.), Radio Engin. Electronic Physics, 1964, 3, 424.

[10] ORAYEvskiy (A. N.), Uspenskiy (A. V.), Trudy, Phys. Inst. Acad. Sci., 1965, 31, 96.

[11] Audorn (C.), C. R. Acad. Sc., 1966, 263, série B, 489.

[12] Fano (U.), Rev. Mod. Physics, 1957, 29, 74.

[13] Grossetête (F.), J. Physique, 1964, 25, 383.

[14] Grossetî̂TE (F.), Thèse, Paris, 1967.

[15] Bender (P. L.), Phys. Rev., 1963, 132, 2154.

[16] Baliming (L. C.), Hanson (R. J.), Pipkin (F. M.), Phys. Rev., 1964, 133, A 607.

[17] BERG (H. C.), Thèse, Harvard, 1964.
[18] Crampton (S. B.), Thèse, Harvard, 1964.

[19] Berg (H. C.), Phys. Rev., 1965, 137, A 1621.

[20] GrossETÊTE (F.), Communication privée.

[21] KLEPPNER (D.), GOLDENBERG (H. M.), RAMSEY (N. F.), Phys. Rev., 1962, 126, 603.

[22] Feynmann (R. F.), Vernon (F. L.), Helimarth (R. W.), J. Appl. Physics, 1957, 28, 49.

[23] Abragam (A.), Les principes du magnétisme nucléaire, P.U.F., Paris, 1961.

[24] Audoin (C.), Schermann (J. P.), C. R. Acad. Sc., 1967, 264, série B, 647.

[25] AudoIn (C.), Thèse, Paris, 1967.

[26] LAMB (W. E.), Quantum Mechanical Amplifiers, dans Lectures on Theoretical Physics, édité par W. E. Brittin et B. W. Downs, Interscience Publishers, N.Y., 1960.

[27] Durand (G.), Thèse, Paris, 1966.

[28] LAMB (W. E.), Phys. Rev., 1964, 134, A 1429.

[29] Audoin (C.), Desaintruscien (M.), SchermanN (J. P.), C. R. Acad. Sc., 1967, 264, série B, 698.

[30] Audoin (C.), C. R. Acad. Sc., 1966, 263, 542.

[31] Audorn (C.), C. R. Acad. Sc., 1967, 264, série B, 385.

[32] VANIER (J.), VessoT (R. F. C.), Quantum Electronics Conference, Phœnix Arizona 19-21, avril 1966.

[33] Audoin (C.), Checkrahamatoula, Onde élect., septembre 1965.

[34] Desaintfuscien (M.), SchermanN (J.-P.), Onde élect., octobre 1966.

[35] AudoIn (C.), Rev. Physique Appliquée, 1966, 1, 2.

[36] Audorn (C.), J. Physique, 1965, 26, 71 A.

[37] Audorn (C.), Deuxième cycle de conférences sur les aimants permanents, Paris, 1965.

[38] Audoin (C.), Septier (A.), Rev. Physique Appliquée, 1966, 1, 95.

Le Directeur de la Publication : Jules-Jean Choviv. — Imprimerie des Presses Universitaires de France, Vendôme (France) Dépôt légal : 1-1968 